\title{
Supporting Information: Dual-color Peak Force Infrared Microscopy
}

\author{
Qing Xie, ${ }^{a}$ Jared Wiemann, ${ }^{\mathrm{b}}$ Yan $\mathrm{Yu},{ }^{\mathrm{b}}$ and Xiaoji G. $\mathrm{Xu}{ }^{\mathrm{a}^{*}}$ \\ ${ }^{a}$ Department of Chemistry, Lehigh University, 6 E Packer Ave., Bethlehem, PA, 18015, United States \\ ${ }^{\mathrm{b}}$ Department of Chemistry, Indiana University, Bloomington, IN 47405, US \\ *E-mail: xgx214@lehigh.edu
}

This supporting information includes:

Figure S1. Two single-frequency PFIR scans of a PS- $b$-PMMA block polymer thin film.

Figure S2. Spatial resolution of Dual-Color PFIR.

Figure S3. Height and width of the outer membrane of $E$. coli bacteria from topography. 

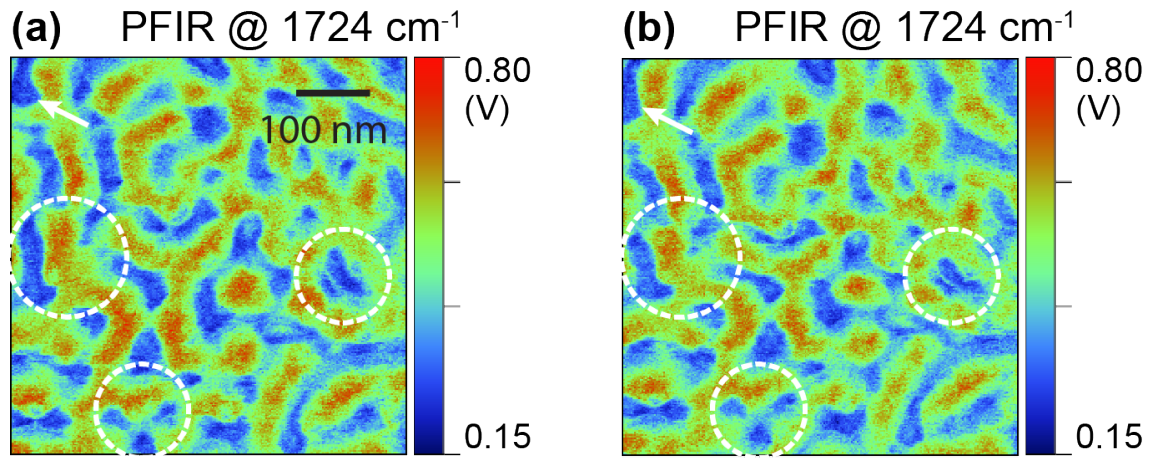

Figure S1. Two single-frequency PFIR scans of a PS- $b$-PMMA block polymer thin film. (a, b) Single-frequency PFIR collected sequentially at $1724 \mathrm{~cm}^{-1}$ under the scan size of 500 $\mathrm{nm} \times 500 \mathrm{~nm}$. The white arrow and dashed circles mark the drift and distort between the two individual scans. 
(a) PFIR@1724 cm-1

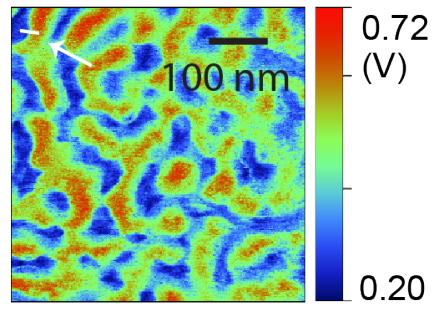

(b) PFIR@1492.7 cm-1

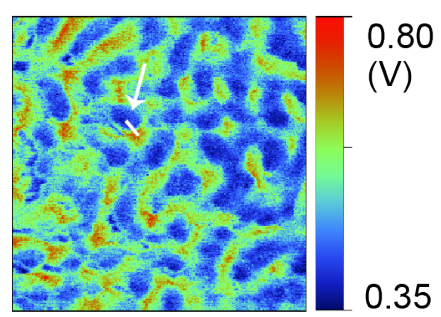

(c) Spatial Resolution

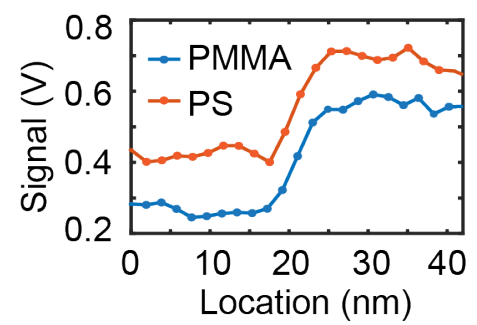

Figure S2. Spatial resolution of Dual-Color PFIR. ( $a, b)$ Dual-Color PFIR images of the PSb-PMMA block copolymer at $1724 \mathrm{~cm}^{-1}$ and $1492.7 \mathrm{~cm}^{-1}$ under the infrared absorption of PMMA and PS, respectively. (c) The spatial resolution is $6 \mathrm{~nm}$ estimated from the width between 10 and $90 \%$ of the edge height of the PFIR signal from both PMMA and PS. The signals are extracted from the section profiles marked with a white line in (a) and (b). 


\section{(a) Topography}

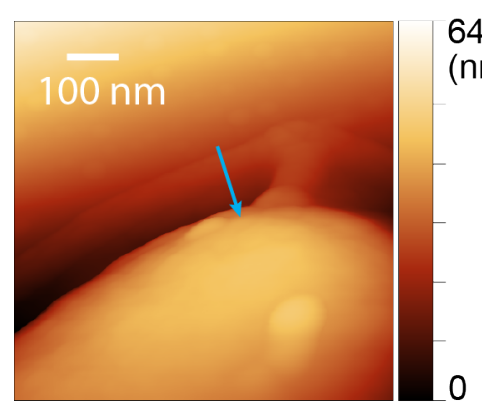

(b) Height Profile

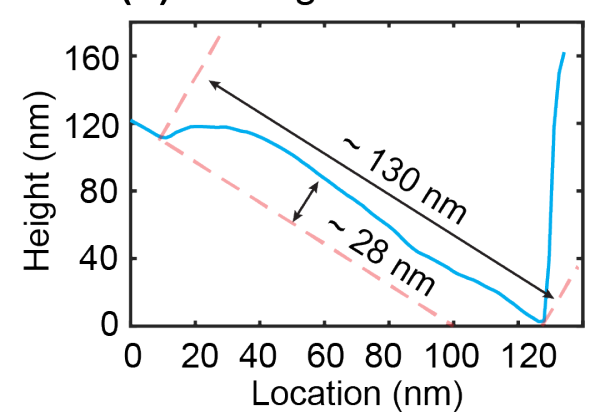

Figure S3. Height and width of ring structure surrounding $E$. coli. (a) Topography of a part of a E coli. (b) Height profile extracted from the blue arrow marked profile on the surrounding ring structure of $E$. coli in (a). Assisted with the pink dashed lines, the height and width of the ring are estimated as $\sim 28 \mathrm{~nm}$ and $\sim 130 \mathrm{~nm}$, respectively. 